\title{
Experiment and Evaluation of Acute Rheumatic Fever Diagnosis Application
}

\author{
Sanjib Raj Pandey \\ Faculty of Architecture, Computing \& Humanities \\ University of Greenwich, London, UK \\ S.R.Pandey@gre.ac.uk \\ Xiaoming Yao \\ College of Information Science and Technology \\ Hainan University, Haikou, China \\ Yaoxiaoming@163.com
}

\author{
Chio-Hong Lai \\ Faculty of Architecture, Computing \& Humanities \\ University of Greenwich, London, UK \\ C.H.Lai@gre.ac.uk \\ Jixin Ma \\ Faculty of Architecture, Computing \& Humanities \\ University of Greenwich, London, UK \\ J.Ma@gre.ac.uk
}

\begin{abstract}
This paper describes an Acute Rheumatic Fever (ARF) Diagnosis Application that is designed and developed based on the proposed Hybrid Approach. It is an integrated framework in terms of a combination of Knowledge-based System, Temporal Theory and Fuzzy Logic. The developed ARF Diagnosis Application was experimentally tested and evaluated by the experts and users of Nepal Heart Foundation (NHF) NHF by means of using NHF's data sets consisting of 676 real patients' records. The ARF Diagnosis Application was found to match 99 percentage of the cases derived from NHF's datasets. The overall ARF diagnostics performance and accuracy was 99.36 percentage.
\end{abstract}

Keywords-Acute Rheumatic Fever; Hybrid Approach; Decision Support System;

\section{INTRODUCTION}

The main purpose of this project to analyse, design and develop a cost effective Clinical Decision Support System (CDSS) for diagnosis and recognition of Acute Rheumatic Fever (ARF) and Rheumatic Heart Disease (RHD) at an early stage by developing/adopting UK's and the Nepal Heart Foundation (NHF)'s treatment practices and procedures that would be appropriate for the Nepalese environment and lifestyle. The system developed is designed for use by community health workers and doctors in the rural areas of Nepal where laboratory facilities, expert services and technology are often deficient.

ARF and RHD are Nepal's biggest medical problems in comparison with other heart diseases. According to Shahid Gangalal National Heart Centre (SGNHC) report of 2007, RHD had the second highest incidence rate with 430 cases [1]. According to the NHF's report, "ARF caused 25-40\% of all cardio-vascular disease in developing countries. Death by RHD is mainly caused by recurrent attacks of ARF." According to the National Heart Centre (NHC), $0.34 \%$ of admitted cases were related to ARF. Recently, this figure increased to $1.3 \%$. All admitted cases of cardiovascular disease were for patients under the age of 18 [1]. The June 2007 - Oct 2011 [2] register of National ARF / RHD Prevention and Control Programme from 32 Hospitals in Nepal, revealed that 6028 ARF / RHD cases had been registered for secondary prevention. These patients were suffering from ARF and were receiving three weekly Benzathine Penicillin G (BPG) injections or oral antibiotics for secondary prevention of ARF [2]. Furthermore, based on the NHF's ARF/RHD registers, during the period of June 2007 to February 2010, from 35 hospitals, 4,712 patients were receiving three weekly injections of BPG, where 2.540 (53.9\%) and 2,172 (46.1\%) were female and male patients respectively. Positive diagnosis of ARF cases numbered 665 (14.1\%) and for RHD there were 4,047 (58.95\%). These figures indicate that all the 4,712 patients suffering from ARF who were unable to obtain treatment for ARF resulted in succumbing to RHD eventually. The $36.7 \%$ of patients were below 18 years and $63.3 \%$ were older than 18 . In a continuous treatment process, $286(6.0 \%)$ of the total patients had missed more than two doses of BPG injection. The reasons for dropout from the BPG injection were phobia of injection pain $(4.95 \%)$, prohibitive distance $(0.8 \%)$, unaffordable cost $(0.2 \%)$ and other $(0.3 \%)$ [3].

\section{A HYBRID APPROCH TO DIAGNOSIS OF ARF}

Currently in Nepal there is no computer-based application designed to help in the diagnosis and management of ARF. Our research aimed to provide better diagnosis of ARF through a computer-based decision support application. The Hybrid Approach aims to apply knowledge-based, rule-based reasoning, which employs guidelines for diagnosing ARF within a Temporal Model. The fuzzy model aims to improve the precision of such diagnosis. ARF Diagnosis Applications would thus be a process for capturing and representing knowledge from experts in order to design and develop an application as well as provide assistance eventually for all users.

The ARF diagnosis process model is divided into three stages: 1) Detected ARF; 2) Suspected ARF and 3) NotDetected ARF [4]. If an ARF case is detected confirming that ARF is present the model should be able to detect the severity level of the case according to observed signs and symptoms after which it will be possible to classify it as a Severe Case, Moderate Case, Mild Case or Suspected Case. If an ARF case 
is suspected then the system will indicate required laboratory testing to acquire further clinical information. If ARF is not confirmed then the findings would likely point towards the possibility of another disease so that the community rural health worker can refer the patient to the nearest hospital or seek the advice of senior doctors if available [4]. Fig. 1 shows the proposed diagnosis model for diagnosis of ARF [4]:

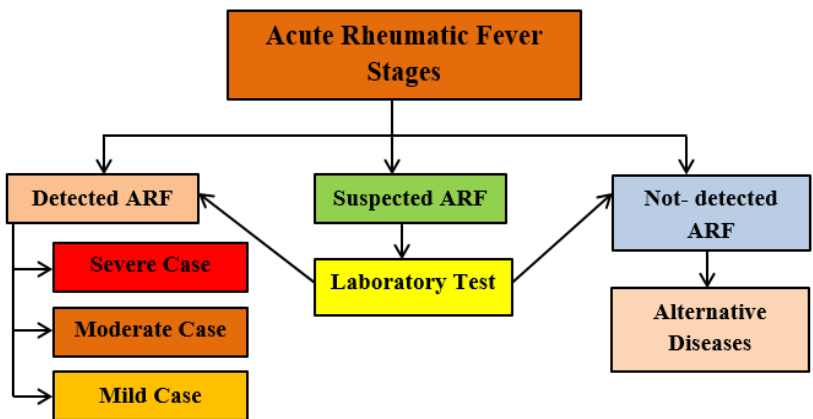

Fig.1. Proposed Diagnosis Model of ARF

The Hybrid processes that are applied in the design and development of an ARF Diagnosis Application are given below :

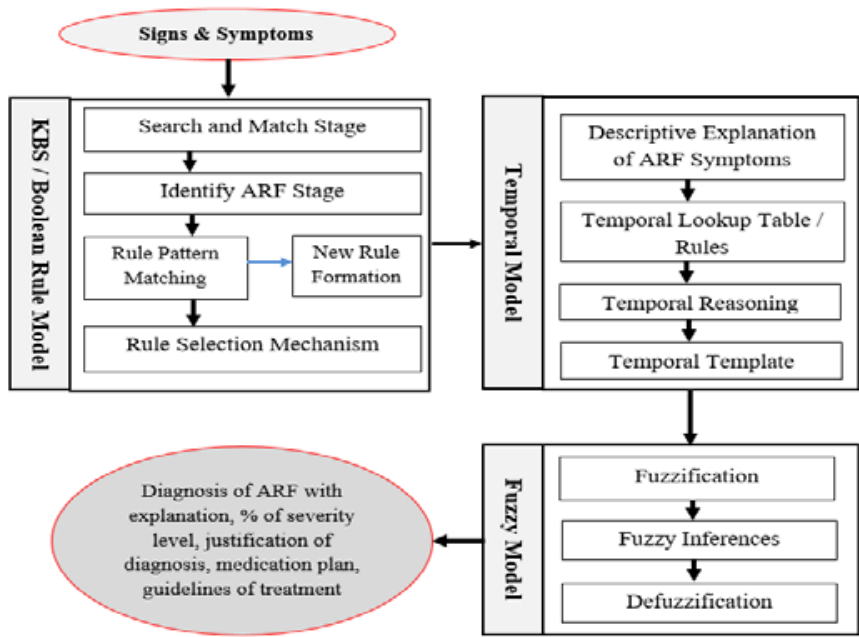

Fig. 2. The Hybrid Process for ARF Diagnosis.

The Hybrid Approach is a combination of the KBS/Boolean Rule Model, Temporal Model and Fuzzy Model. The KBS/Boolean Rule Model has four components for design and implementation of KBS. These are: identifying the ARF stage in a case; Rule Pattern Matching; New Rule Formation and Rule Selection Mechanism. This model is used for capture, management and representing ARF knowledge as well as for the construction of the Boolean rules. The second model used is Temporal and is responsible for capturing the event and time from a patient's description (in his/her native language) during the explanation of symptoms. To simplify this task for doctors or rural health workers, we apply a standard electronic format that selects symptoms and the auto or manual method (user choice) will apply to capture the duration of symptoms. The Temporal Model has four components namely: Descriptive Explanation of ARF symptoms; Temporal Lookup-Table/Rules and Temporal Reasoning, which produce a Temporal Template for demonstrating the relationship between the signs and ARF. The third model is Fuzzy, which is responsible for managing uncertainties, vagueness of signs and symptoms and for making a final diagnosis of ARF. Fuzzification, Fuzzy Inference and Defuzzification are components of this model [6] [7]. The research undertaken divided the overall ARF diagnosis problem, in effect its requirements, into several subproblems and each model of the Hybrid Approach addressed particular sub-problems for example, Identify the stage of the ARF component of the KBS/Boolean Rule Model used to solve the question of identifying the stage of ARF based on the symptoms presented. Each problem was therefore handled using a particular model's components. This significantly helped to improve maintainability, reliability and the overall quality of our final ARF Diagnosis Application. Further details about the model development can found on [5] [7] and our conceptual framework can found on [4].

\section{EXPERIMENT AND EVALUATION}

The objectives of our experiments and evaluation were to test and validate the following key areas of the ARF diagnosis Application as given below:

1. The developed ARF Diagnosis Application meets the predefined NHF requirements or not.

2. Evaluate whether the proposed Hybrid Approach and its components are appropriate for the development of an ARF Diagnosis Application for Nepal or not.

3. For the proposed ARF Diagnosis Application's usability, evaluate whether the application can be used by community rural health workers and inexperienced doctors and check how ARF Diagnosis Applications can handle ARF ambiguities.

The experiment and evaluation of our ARF Diagnosis Application took place in the head office of NHF, Lalitpur, Nepal. The following points were set up for the experiment and evaluation:

- $\quad$ Set up the evaluation criteria.

- Evaluation of functionality, usability, accuracy, efficiency and performance of the ARF Application.

- Test the requirements of the Application.

- Test the Hybrid Approach.

- $\quad$ Testing of the User Interface.

- Validity of the ARF Diagnosis Application using NHF real data sets.

\section{A. ARF Diagnosis Application : Comparison and Result}

The comparison of ARF Diagnosis Application's result using NHF survey datasets are shown in Table I and overall match analyses are provided in Table II. 
TABLE I. COMPARISON RESULT

\begin{tabular}{|l|l|c|c|}
\hline Descriptions & $\begin{array}{c}\text { NHF registry } \\
\text { data set's } \\
\text { result }\end{array}$ & $\begin{array}{c}\text { ARF Diagnosis } \\
\text { Application's } \\
\text { Result }\end{array}$ & $\begin{array}{c}\text { Differences } \\
\text { in number }\end{array}$ \\
\hline $\begin{array}{l}\text { Detected ARF } \\
\text { Cases }\end{array}$ & $87(13.68 \%)$ & $84(13.21 \%)$ & 3 \\
\hline $\begin{array}{l}\text { Suspected } \\
\text { ARF Cases }\end{array}$ & $74(11.64 \%)$ & $73(11.48 \%)$ & 1 \\
\hline $\begin{array}{l}\text { Not Detected } \\
\text { Cases }\end{array}$ & $475(74.69 \%)$ & $479(75.31 \%)$ & 4 \\
\hline Total & $\mathbf{6 3 6}$ & $\mathbf{6 3 6}$ & $\mathbf{8}$ \\
\hline
\end{tabular}

Based on the total 636 NHF survey datasets, 87 (13.68\%) patients were recorded as Detected ARF cases; 74 (11.64\%) patients were recorded as Suspected cases and 475 (74.69\%) patients were recorded as Not detected. Similarly, 636 NHF' survey datasets were tested with our ARF Diagnosis Application. The result revealed that, 84 (13.21\%) patients were identified as Detected cases; 73 (11.48\%) patients were identified as Suspected and 479 (75.31\%) patients identified as Not detected cases. There were only very few difference and Table II shows the comparison results in percentages.

TABLE II. COMPARISON RESULT IN PERCENTAGE

\begin{tabular}{|l|l|c|c|}
\hline \multicolumn{1}{|c|}{ Descriptions } & \multicolumn{1}{|c|}{$\begin{array}{c}\text { Matching } \\
\text { with NHF's } \\
\text { data set }\end{array}$} & $\begin{array}{c}\text { Not matching } \\
\text { with NHF data }\end{array}$ & Total \\
\hline $\begin{array}{l}\text { Detected ARF } \\
\text { Cases }\end{array}$ & $97 \%$ & $84(13.21 \%)$ & $100 \%$ \\
\hline $\begin{array}{l}\text { Suspected ARF } \\
\text { Cases }\end{array}$ & $99 \%$ & $73(11.48 \%)$ & $100 \%$ \\
\hline $\begin{array}{l}\text { Not Detected } \\
\text { Cases }\end{array}$ & $99 \%$ & $1 \%$ & $100 \%$ \\
\hline
\end{tabular}

Table III shows the overall comparison (match and not match cases of NHF ARF datasets) and results of our ARF Application using NHF datasets.

TABLE III. OVERALL COMPARISON RESULT WITH NHF DATASETS

\begin{tabular}{|r|r|}
\hline No. of cases matches with NHF dataset & $\mathbf{6 2 3}$ \\
\hline No. of difference case (not matches cases) : & 8 \\
\hline \% of matches Case : & $98.74 \%$ \\
\hline \% of not matches Case : & $1.26 \%$ \\
\hline Total & $100 \%$ \\
\hline
\end{tabular}

The accuracy of our ARF Diagnosis Application is 98.74\%. In the difference cases, the number of patients had already been diagnosed with heart disease and was taking medicine. These differences were revealed in the comparison result. This research did not consider the previous history of ARF and RHD diseases. Fig. 3. shows comparison results of Detected, Suspected and Not detected cases.

\section{Comparison Result}

जHF registry data sets result $\quad$ ARF diagnosis application's result

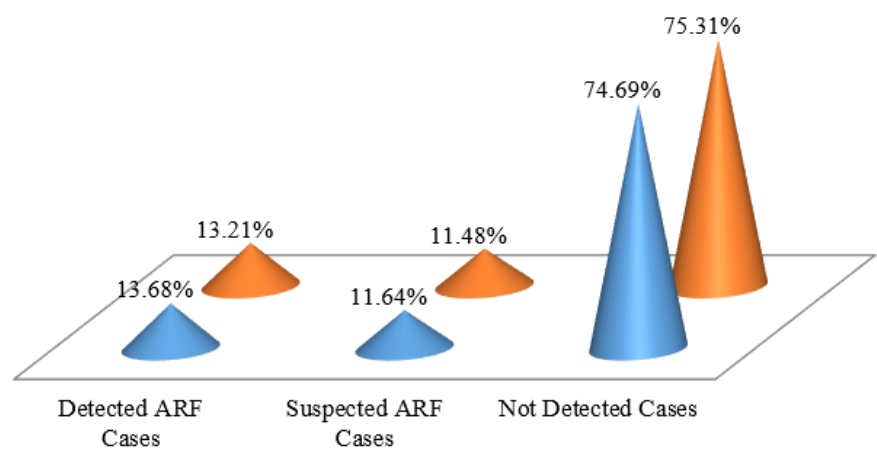

Fig.3. Comparison Result Chart

\section{B. Experiment and Evaluation of ARF Application Quality and Accuracy}

The quality and accuracy of the identification of ARF stages from our Application revealed $75 \%$ of experts were very satisfied and $25 \%$ were satisfied. The identification of ARF stages was a key component of our ARF Diagnosis Application. For this, $100 \%$ of NHF experts were satisfied over the fact that production and definition of identification stages by our ARF Application were correct.

\section{Experimentand Evaluation of User Interfaces}

The Heuristic method is used for software usability evaluation. Evaluators will inspect the interface and identify any inherent problems [8]. Our ARF's Diagnosis Application's interface was examined by NHF's expertise from the outset. A set of questions was answered by 9 users including NHF's experts (4), community rural health workers (3), NHF's IT expert internal (1), and IT expert external (1). The following Fig. 5. shows the user ARF Diagnosis Application's User Interface evaluation results in a column chart.

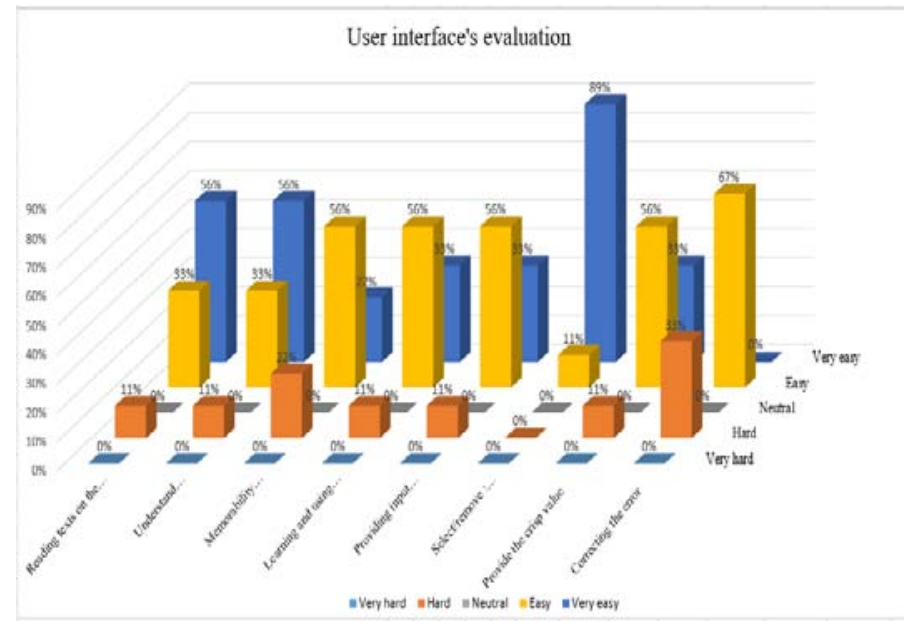

Fig. 4. User Interface Evaluation Result. 


\section{Experimentand Evaluation of Application's Information and Message}

The ARF Diagnosis Application's information (help and supporting file information) pattern (text, audio, picture, Nepali and English language) and message (error message, warning message) evaluation results are shown in Fig. 5.

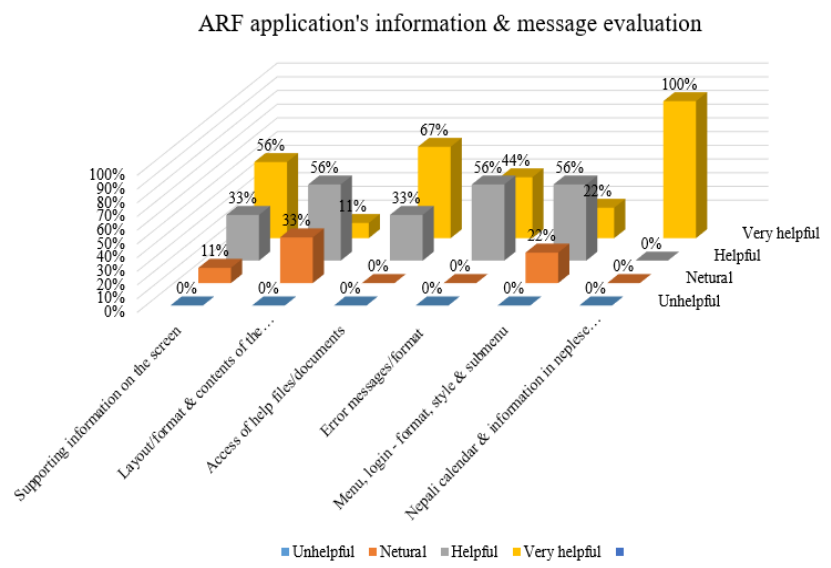

Fig. 5. ARF ARF Application's Information and Message Evaluation.

\section{CONCLUSION}

The Application has been evaluated with NHF's survey datasets (636 records). Comparison of result shows that in total $98.74 \%$ matched with NHF's datasets and $1.26 \%$ did not match. The quality and accuracy of the methods, processes and functionalities of the Application were tested and evaluated by NHF experts. The accuracy of the identification of ARF stages was as follows: $75 \%$ were very satisfied and $25 \%$ were satisfied (100\% satisfied overall). All participants who were surveyed stated that they were very satisfied with the accuracy and quality of our New Rule Formation process. So, in general $100 \%$ were satisfied that our Application was developed properly in accordance with their requirements. The overall accuracy of our ARF Diagnosis Application was found to be $99.36 \%$.

Our ARF Diagnosis Application was thus successfully developed, experimented and evaluated meeting NHF's diagnosis criteria for treatment of ARF. The output of the evaluation was proof that the ARF Diagnosis Application we had developed in our collaboration programme met the NHF's requirements. During experimentation and evaluation the following facts were identified as a key indicators and points for success of our ARF Diagnosis Application:

- Presented an interest and commitment of NHF's experts and users regarding the use of our innovative ARF Diagnosis Application.

- The Application's interface is user friendly.

- ARF Diagnosis Application suits the Nepalese environment and lifestyles.
- ARF knowledge, Temporal Template, Rules and other supporting materials can be updated easily.

- NHF application's supporting information was in both languages (Nepalese and English).

- Our ARF Diagnosis Application will be fully incorporated within the NHF diagnosis criteria since ARF diagnosis decisions are clear and transparent.

- The application very well met the set of criteria established by the NHF.

- The Knowledge-based model can handle effectively the identification of ARF stages, Rule Pattern Matching, Rule Selection Mechanism and New Rule Formation.

- The quality and accuracy of New Rule Formation matched the set guidelines of NHF, and the Rule Selection Mechanism was shown able to select relevant rules from rule-based systems.

- The Temporal Lookup Table and temporal reasoning process can improve the accuracy and quality of ARF diagnostics.

- The fuzzy process can handle effectively all uncertainties pertaining to ARF symptoms.

Taken overall, it was shown that the proposed Hybrid Approach can provide a reliable tool for diagnosis of ARF cases.

\section{References}

[1] SGNHC (2007), 'Annual Report', Shahid Gangalal National Health Centre, Nepal, Available at: http://www.sgnhc.org.np/report2007/main\%20Page\%201\%20to\%2066.pdf. [Accessed: 27 Feb. 2015].

[2] Regmi P R, et al (2012), 'Prevalence of Sydenham's Chorea in Patients with Acute Rheumatic Fever in Nepal', Nepalese Heart Journal, vol.9, No.1, pp 30-32.

[3] Nepal Heart Foundation, unpblished data 2013.

[4] Sanjib Pandey, Ma Jixin and Lai Choi-Hong (2015), 'A Conceptual Framework to Diagnosis of Acute Rheumatic Fever Based on the Temporal and Fuzzy Logic Approach', Canadian International Journal of Science and Technology, vol. 2, pp 397-426 (print), The AngloEgyptian Bookshop, ISBN: 2356-9085.

[5] Sanjib Raj Pandey, Jixin Ma and Chong Hong Lai (2015), 'Development of Temporal Logic-Based Fuzzy Decision Support System for Diagnosis of Acute Rheumatic Fever/Rheumatic Heart Disease', Research and Development in Intelligent Systems XXXII, Springer International Publishing, pp 213-228, 2015, 978-3-319-25030-4, DOI :10.1007/978-3319-25032-8_17.

[6] Pandey Sanjib, Ma Jixin and Lai Choi-Hong (2014) "Fuzzy Membership Function and Input / Output Parameter for Diagnosis of Rheumatic Fever", International Transactions on Information Science and Technology (ITIST), 2 (6), pp.10-15. ISSN 2325-6567 (Print), 23256575

[7] Pandey Sanjib, Ma Jixin and Lai Choi-Hong (2015) "Development of Decision Support System for the Diagnosis of Arthritis Pain for Rheumatic Fever Patients: Based on the Fuzzy Approach” Journal of Algorithms and Computational Technology, Sep 2015, Vol. 9, Issue 3, pp. 265-290, DOI: http://dx.doi.org/10.1260/1748-3018.9.3.265

[8] Zhang J, Johnson TR, Patel VL, Paige DL, Kubose T.(2003), 'Using usability heuristics to evaluate patient safety of medical devices', $J$ Biomed Inform, vol. $36 \quad$ (1-2), pp. 23-30. 